\title{
Thermo-physical properties of 162173 (1999 JU3), a potential flyby and rendezvous target for interplanetary missions (Research Note)
}

\author{
T. G. Müller ${ }^{1}$, J. Ďurech ${ }^{2}$, S. Hasegawa ${ }^{3}$, M. Abe $^{3}$, K. Kawakami ${ }^{3}$, T. Kasuga ${ }^{4}$, D. Kinoshita ${ }^{5}$, D. Kuroda ${ }^{6}$, S. \\ Urakawa $^{7}$, S. Okumura ${ }^{7}$, Y. Sarugaku ${ }^{8}$, S. Miyasaka ${ }^{9}$, Y. Takagi ${ }^{10}$, P. R. Weissman ${ }^{11}$, Y.-J. Choi ${ }^{12}$, S. Larson ${ }^{13}$, \\ K. Yanagisawa ${ }^{6}$, and S. Nagayama ${ }^{14}$ \\ 1 Max-Planck-Institut für extraterrestrische Physik, Giessenbachstraße, 85748 Garching, Germany \\ e-mail: tmueller@mpe.mpg.de \\ 2 Astronomical Institute, Faculty of Mathematics and Physics, Charles University in Prague, V Holešovičkách 2, 180 00, Praha 8, \\ Czech Republic \\ 3 Institute of Space and Astronautical Science, Japan Aerospace Exploration Agency, 3-1-1 Yoshinodai, Sagamihara, Kanagawa \\ 229-8510, Japan \\ 4 Department of Physics and Astronomy, The University of Western Ontario, 1151 Richimond St. London, ON, N6A 3K7, Canada \\ 5 Institute of Astronomy, National Central University, 300 Jhongda RD., Jhongli, Taoyuan 32001, Taiwan \\ ${ }^{6}$ Okayama Astrophysical Observatory, National Astronomical Observatory of Japan, 3037-5 Honjo, Kamogata, Asaguchi, Okayama \\ 719-0232, Japan \\ 7 Bisei Spaceguard Center, Japan Spaceguard Association, 1716-3 Okura, Bisei, Ibara, Okayama 714-1411, Japan \\ 8 Kiso Observatory, Institute of Astronomy, The University of Tokyo, 10762-30 Mitake, Kiso, Nagano 397-0101, Japan \\ 9 Tokyo Metropolitan Government, 2-8-1 Nishishinjyuku, Shinjyuku, Tokyo 163-8001, Japan \\ 10 Aichi Toho University, 3-11 Heiwagaoka, Meito, Nagoya, Aichi 468-8515, Japan \\ 11 Jet Propulsion Laboratory, 4800 Oak Grove Drive, MS 183-301, Pasadena, CA 91109, USA \\ 12 Korea Astronomy and Space Science Institute, 61-1 Hwaam-dong, Yueing-gu, Daejeon, Taejon 305-348, Korea \\ 13 Lunar and Planetary Laboratory, University of Arizona, Tucson, AZ, 85721-0092, USA \\ 14 National Astronomical Observatory of Japan, 2-21-1 Osawa, Mitaka, Tokyo 181-8588, Japan
}

Received 17 August 2010 / Accepted 5 November 2010

\section{ABSTRACT}

Context. Near-Earth asteroid 162173 (1999 JU3) is a potential flyby and rendezvous target for interplanetary missions because of its easy-to-reach orbit. The physical and thermal properties of the asteroid are relevant for establishing the scientific mission goals and also important in the context of near-Earth object studies in general.

Aims. Our goal was to derive key physical parameters such as shape, spin-vector, size, geometric albedo, and surface properties of 162173 (1999 JU3).

Methods. With three sets of published thermal observations (ground-based $N$-band, Akari IRC, Spitzer IRS), we applied a thermophysical model to derive the radiometric properties of the asteroid. The calculations were performed for the full range of possible shape and spin-vector solutions derived from the available sample of visual lightcurve observations.

Results. The near-Earth asteroid 162173 (1999 JU3) has an effective diameter of $0.87 \pm 0.03 \mathrm{~km}$ and a geometric albedo of $0.070 \pm 0.006$. The $\chi^{2}$-test reveals a strong preference for a retrograde sense of rotation with a spin-axis orientation of $\lambda_{\mathrm{ecl}}=73^{\circ}$, $\beta_{\text {ecl }}=-62^{\circ}$ and $P_{\text {sid }}=7.63 \pm 0.01 \mathrm{~h}$. The most likely thermal inertia ranges between 200 and $600 \mathrm{~J} \mathrm{~m}^{-2} \mathrm{~s}^{-0.5} \mathrm{~K}^{-1}$, about a factor of 2 lower than the value for 25143 Itokawa. This indicates that the surface lies somewhere between a thick-dust regolith and a rock/boulder/cm-sized, gravel-dominated surface like that of 25143 Itokawa. Our analysis represents the first time that shape and spin-vector information has been derived from a combined data set of visual lightcurves (reflected light) and mid-infrared photometry and spectroscopy (thermal emission).

Key words. minor planets, asteroids: individual: 162173 (1999 JU3) - radiation mechanisms: thermal - techniques: photometric infrared: planetary systems

\section{Introduction}

Asteroid 162173 (1999 JU3) is currently among the potential targets of future interplanetary exploration missions. The target is relatively easy to reach with state-of-the-art mission capabilities, and it offers high scientific potential (Binzel et al. 2004). This near-Earth asteroid belongs to the C-class objects, which are believed to represent primitive, volatile-rich remnants of the early solar system. Various aspects of this small body have been covered in some detail in the recent works by Hasegawa et al. (2008) and by Campins et al. (2009).

Hasegawa et al. (2008) use a spherical shape model for their radiometric analysis, and alternatively use an ellipsoidal shape model, but without knowing the true spin-vector orientation. The results (radiometric diameter of $0.92 \pm 0.12 \mathrm{~km}$, visual geometric albedo of $0.063_{-0.015}^{+0.020}$ ) which indicate a thermal inertia larger than $500 \mathrm{~J} \mathrm{~m}^{-2} \mathrm{~s}^{-0.5} \mathrm{~K}^{-1}$ ), were based on a set of photometric 
Subaru and Akari observations and connected to simplified shape and spin-axis assumptions. Campins et al. (2009) have obtained a single-epoch Spitzer infrared spectrograph (IRS) spectrum. They used a spherical shape model, and for the spin-pole orientation they used the extreme case of an equatorial retrograde geometry and a prograde solution published by Abe et al. (2008). Their analysis, based on the single IRS-spectrum and ignoring the data sets published by Hasegawa et al. (2008), yielded a value for the thermal inertia of $700 \pm 200 \mathrm{~J} \mathrm{~m}^{-2} \mathrm{~s}^{-0.5} \mathrm{~K}^{-1}$, a diameter estimate of $0.90 \pm 0.14 \mathrm{~km}$, and geometric albedo of $0.07 \pm 0.01$.

Despite the simplifications in shape and spin-vector properties, both sets of published radiometric diameter and albedo values agree within the given uncertainties. Both teams also favoured a relatively high thermal inertia (close to that of 25143 Itokawa). The unknowns of the spin-vector orientation cause a large uncertainty in the thermal properties.

Here we re-analyse all available lightcurve observations to derive (on the basis of standard $\chi^{2}$ lightcurve inversion techniques) all matching spin-vector and shape solutions (Sect. 2). The full possible range for shape, spin-axis orientation, and rotation period was then used as input for a thermophysical $\chi^{2}$ analysis of all available thermal observations (Sect. 3) with the goal of deriving radiometric sizes, albedos and thermal inertias. At the same time, we determined the most likely shape-solution, rotation period and spin-axis orientation (Sect. 4).

\section{Possible shape and spin-vector solutions}

A detailed list of the available photometric observations is presented in Table 1 . There are about 40 dedicated visual lightcurve data sets spread over more than 270 days. About half of the lightcurves were calibrated; some of the data sets are very noisy. Based on these data, Abe et al. (2008) found a rotation period of $7.6272 \pm 0.0072 \mathrm{~h}$ and a spin orientation of $\lambda_{\mathrm{ecl}}=331.0^{\circ}$, $\beta_{\mathrm{ecl}}=+20.0^{\circ}$, indicating a prograde rotation. A more recent analysis by the same authors (priv. communication) resulted in a rotation model with slightly different values: $\lambda_{\mathrm{ecl}}=327.3^{\circ}$, $\beta_{\text {ecl }}=+34.7^{\circ}, P_{\text {sid }}=7.6273922 \mathrm{~h}$. Both solutions were derived using the epoch and amplitude methods described by Magnusson (1986). These methods are reliable for irregularly shaped bodies and for sufficient lightcurve data covering various aspect angles. 1999 JU3 has a comparatively spherical shape and the available lightcurve data were from restricted directions. Nevertheless, both solutions gave a reasonable match to the observed lightcurves, but it turned out that these solutions are not unique and other parameter sets with different rotation periods, spin-axis orientations and shape models could not be excluded (see Fig. 1).

Since these values are crucial input parameters for our thermophysical model analysis, we repeated the search for possible shape and spin-vector solutions using the lightcurve inversion method developed by Kaasalainen \& Torppa (2001). Our goal was to derive a set of the most likely convex shape models that would fit all available lightcurves. Because of the poor quality of some of the data, we were only able to determine the range of the sidereal rotation period to 7.6204-7.6510 h. Different shape models with pole orientations covering almost the entire celestial sphere (without any preference for pro- or retrograde solutions) fit the data equally well. By scanning the period-pole parameter space, we derived 77 shape models that were physically acceptable (rotating around the shortest axis) and for which the $\chi^{2}$ of the fit was no more than $10 \%$ higher than the best-fit $\chi^{2}$. These models corresponded to local minima in the parameter space.
Table 1. Observation circumstances for the lightcurve measurements.

\begin{tabular}{lll}
\hline \hline Mon/Day (2007) & Telescope & Observer \\
\hline $07 / 8,09 / 4$ & $2.2 \mathrm{~m} /$ Mauna Kea & T. Kasuga \\
$07 / 19-23,12 / 3,4,6-8$, & $1.0 \mathrm{~m} /$ Lulin & M. Abe, K. Kawakami, \\
$02 / 26-28,04 / 2,4,5$ & & D. Kinoshita \\
$08 / 5,15,09 / 6,11,13,15,1.0 \mathrm{~m} /$ Ishigaki & D. Kuroda, S. Nagayama, \\
10/6,18, 11/13,15 & & K. Yanagisawa \\
$08 / 9-10,17,20,09 / 6,10$ & $1.0 \mathrm{~m} /$ Bisei & S. Urakawa, \\
& & S. Okumura \\
$09 / 4,5,7,8,10,12,14,15$ & $1.05 \mathrm{~m} /$ Kiso & M. Abe, K. Kawakami, \\
$11 / 7-9,11,13,02 / 5-8$, & & Y. Sarugaku, Y. Takagi, \\
$04 / 14,15$ & \multirow{2}{*}{$1.55 \mathrm{~m} /$ Steward } & S. Miyasaka \\
$09 / 11-14$ & & P. R. Weissman, \\
& & Y.-J. Choi, S. Larson \\
\hline
\end{tabular}

Notes. See also Table 1 in Abe et al. (2008).
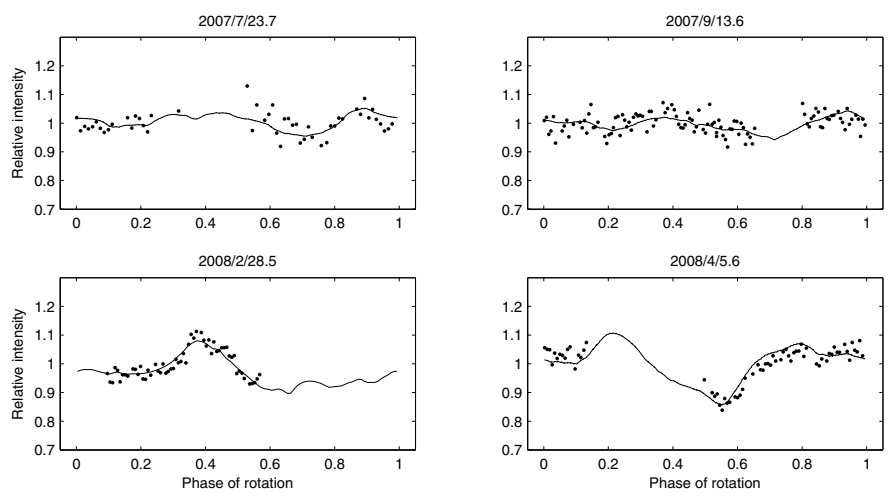

Fig. 1. Match between observed lightcurves and shape/spin-axis model solution "7_1".

In addition, we included both of the original Abe et al. (2008) solutions and added another five with the pole fixed to the two $\left(\lambda_{\mathrm{ecl}}, \beta_{\mathrm{ecl}}\right)$-pairs mentioned above and rotation periods in the range given by Abe et al. (2008). Only two of these models pass the $\chi^{2}+10 \%$ limit on the basis of the visual lightcurves, the other five models have higher $\chi^{2}$-values. Six of these shape models are elongated along the " $z$ " axis and therefore unphysical.

For all 84 models we performed a thermophysical model analysis for a wide range of possible parameters (see Table 3).

\section{Thermophysical model analysis}

\subsection{Model and input parameters}

The mid-IR photometric data were already described in Hasegawa et al. (2008). The data set includes $15 \mathrm{~N}$-band Subaru observations and two dedicated Akari observations at 15 and $24 \mu \mathrm{m}$. We binned the single-epoch Spitzer IRS data (Campins et al. 2009) into 20 wavelength points ( 4 for band SL2, 7 for SL1, 4 for LL2, 5 for LL1; see Fig. 5, bottom). The 20 wavelength points were chosen to give about equal weight to the two published data samples in terms of number of observations (17 in Hasegawa et al. (2008) and 20 for the Campins et al. (2009) sample). In this way the derived object properties are better connected to the entire data set, and they do not just match the measurements of one observing epoch. Each observation set also has a mixture of lower and higher quality data: the ground-based Subaru data are of lower quality than the Akari data, while the quality of the IRS spectrum changes with wavelength. This again 
Table 2. Summary of the avaible thermal observations.

\begin{tabular}{lllllll}
\hline \hline Year/Mon/Day & Wavelength range $[\mu \mathrm{m}]$ & $R_{\mathrm{h}}[\mathrm{AU}]$ & $\Delta[\mathrm{AU}]$ & $\alpha\left[^{\circ}\right]$ & Telescope & Reference \\
\hline $2007 / 05 / 16$ & $15.0,24.0$ & 1.414 & 0.992 & +45.6 & Akari & Hasegawa et al. (2008) \\
$2007 / 08 / 28$ & $8.8(3 \times), 9.7(1 \times), 10.5(1 \times), 11.7(7 \times), 12.4(3 \times)$ & 1.287 & 0.306 & +22.3 & Subaru & Hasegawa et al. (2008) \\
$2008 / 05 / 02$ & $5.2-8.5,7.4-14.2,14.0-21.5,19.5-38.0$ & 1.202 & 0.416 & $+52.6^{\circ}$ & Spitzer & Campins et al. (2009) \\
\hline
\end{tabular}

Notes. $R_{\mathrm{h}}$ is the helio-centric distance and $\Delta$ the distance between object and telescope. All observations were taken at positive phase angles $\alpha$ (Sun-object-telescope), i.e., leading the Sun, with a cold terminator for a retrograde rotating body.

Table 3. Summary of general TPM input parameters and applied ranges.

\begin{tabular}{lcl}
\hline \hline Param. & Value/Range & Remarks \\
\hline$\Gamma$ & $0 \ldots 2500$ & $\mathrm{~J} \mathrm{~m}^{-2} \mathrm{~s}^{-0.5} \mathrm{~K}^{-1}$, thermal inertia \\
$\rho$ & $0.4 \ldots 0.9$ & rms of the surface slopes \\
$f$ & $0.4 \ldots 0.9$ & surface fraction covered by craters \\
$\epsilon$ & 0.9 & emissivity \\
$H_{\mathrm{V}}$-mag. & $18.82 \pm 0.02 \mathrm{mag}$ & Abe et al. $(2008)$ \\
G-slope & $-0.110 \pm 0.007$ & Abe et al. $(2008)$ \\
shape & 84 models & see Sect. 2 \\
spin-axis & 84 solutions & see Sect. 2 \\
$P_{\text {sid }}[\mathrm{h}]$ & $7.6205 \ldots 7.6510$ & see Sect. 2 \\
\hline
\end{tabular}

ensures that the final solutions are not biased towards a single measurement. All observations are listed in Table 2.

All 84 possible spin-vector and shape solutions from Sect. 2 have been used in combination with these thermal data.

For our analysis we are using a thermophysical model (TPM) described by Lagerros (1996, 1997, 1998) and Müller \& Lagerros (1998). This TPM works with true illumination and observing geometries, accepts irregular shape models and arbitrary spin-vector solutions, works with roughness controlled by the rms of the surface slopes, considers heat-conduction into the surface as well as multiple scattering of both the solar and the thermally emitted radiation. The model has been tested and validated thoroughly for NEAs (e.g., Müller et al. 2005) and MBAs (e.g., Müller \& Lagerros 2002). The TPM input parameters and applied variations are listed in Table 3.

\subsection{Solving for effective diameter, geometric albedo and thermal inertia}

Campins et al. (2009) and Mueller (2007) used a $\chi^{2}$ or reduced $\chi^{2}$-test to find solutions for the thermal inertia $\Gamma$. Here we follow a modified approach to find the most robust solutions with respect to thermal inertia and allowing for the full range in effective diameter and geometric albedo at the same time. The following procedure was executed for all 84 possible shape and spin-vector solutions separately:

(1) For each value of $\Gamma$ in a wide range (see Table 3 ) we calculate the radiometric diameter and albedo solution via the TPM for each individually observed thermal flux (37 individual diameter and albedo solutions). Diameter and albedo are linked by the absolute magnitude $H_{\mathrm{V}}$ which was kept constant (the rotational amplitude is only about $0.1 \mathrm{mag}$ ).

(2) We calculate the weighted mean radiometric diameter and albedo solution for each given $\Gamma\left(\bar{x}=\frac{\Sigma x_{i} / \sigma_{i}^{2}}{\Sigma 1 / \sigma_{i}^{2}}\right.$, with diameter/albedo errors $\sigma_{i}$ connected to the observational errors).

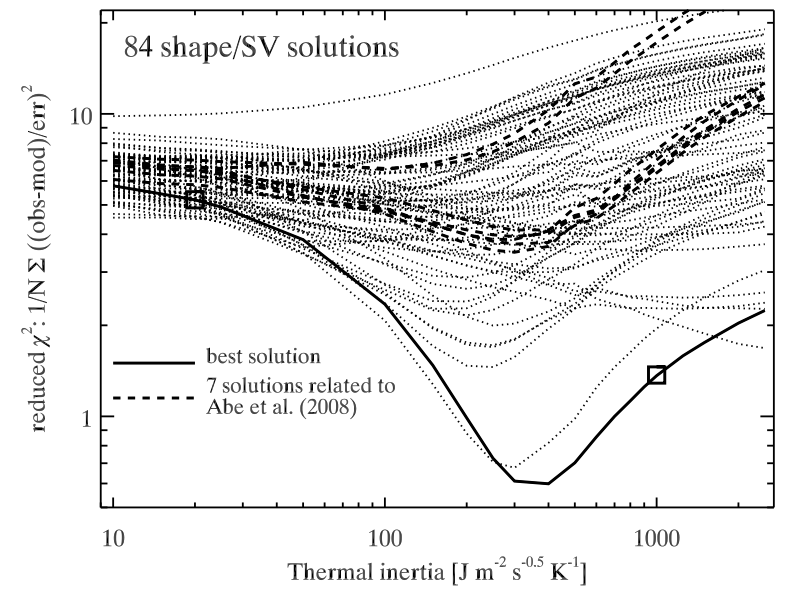

Fig. 2. TPM $\chi^{2}$-optimization process to find robust solutions for diameter, albedo and thermal inertia simultaneously. Each line represents the reduced $\chi^{2}$ values for an individual shape/spin-vector solution as a function of thermal inertia. The surface roughness was kept constant using the "default values" of $\rho=0.7$ and $f=0.6$ as proposed by Müller et al. (1999). Model 7_1 solutions marked with squares on the solid line correspond to the two cases with thermal inertias of 20 and $1000 \mathrm{~J} \mathrm{~m}^{-2} \mathrm{~s}^{-0.5} \mathrm{~K}^{-1}$ shown in Fig. 3 (left and middle).

(3) For each individual observation we predict TPM fluxes based on the given $\Gamma$ and the corresponding weighted mean radiometric diameter and albedo from step (2).

(4) The most robust solutions occur when the observations and the TPM predictions agree best (taking the uncertainties of the measurements into account in a weighted mean sense, see step 2). This can be expressed as $\frac{1}{N} \Sigma_{i=1}^{N}\left(\left(\mathrm{obs}_{i}-\bmod _{i}\right) / \sigma_{i}\right)^{2}$, a modified reduced $\chi^{2}$ method. The most likely thermal inertia is found at the smallest $\chi^{2}$ values; the connected effective diameter and geometric albedo values are the ones calculated at step (2).

In a first round of executing this procedure we kept the surface roughness constant at values which were specified as "default values" for large, regolith-covered asteroids (Müller et al. 1999). The corresponding roughness parameter values are $\rho=0.7$, the rms of the surface slopes, and $f=0.6$, the fraction of surface covered by craters. The results are shown in Fig. 2.

The best shape and spin-vector solutions (with lowest values for the reduced $\chi^{2}$ and clear minima in Fig. 2) were then the starting point for further tests:

(i) Are these solutions robust against sub-sets of the thermal data?

(ii) How does the surface roughness influence the solutions?

(iii) Do the solutions explain the thermal behaviour over the observed phase angle range (from $\sim 20^{\circ}$ to $\sim 55^{\circ}$ )? 
Table 4. The shape and spin-vector solutions which produce the lowest $\chi^{2}$-values in Fig. 2.

\begin{tabular}{lllll}
\hline \hline model-ID & $\lambda_{\text {ecl }}\left[{ }^{\circ}\right]$ & $\beta_{\text {ecl }}\left[^{\circ}\right]$ & $P_{\text {sid }}[\mathrm{h}]$ & $\chi^{2}-\min$ \\
\hline $7 \_1^{a}$ & 73.1 & -62.3 & 7.6323 & 0.60 \\
$8 \_2^{a}$ & 69.6 & -56.7 & 7.6325 & 0.68 \\
$14 \_8$ & 77.1 & -30.9 & 7.6510 & 1.46 \\
\hline
\end{tabular}

Notes. ${ }^{(a)}$ Models 7_1 and $8 \_2$ are in the same local minimum in the parameter space for the lightcurve fits.

(iv) Is the TPM match of equal quality at all observed wavelengths?

(v) Are there large discrepancies at certain rotational phases?

\section{Results and discussion}

\subsection{Solution for shape and spin-vector}

The shape and spin-vector solutions which produce the lowest $\chi^{2}$-values in Fig. 2 are listed in Table 4. The model IDs represent a full shape-model, each with more than 2000 surface elements and more than 1000 vertices. The Julian date at zero rotational phase $\gamma_{0}$ is in all cases $T_{0}=2454289.0$.

All three solutions are retrograde solutions and, in fact, the eight best solutions in Fig. 2 are retrograde solutions. The best prograde solutions in the $\chi^{2}$ picture are models with ID 5_2 and ID 13_5, both have $\chi^{2}$-minima at 2.3 (almost a factor of 4 higher than the best retrograde solution 7_1) and would require an extremely high thermal inertia $\left(>1000 \mathrm{~J} \mathrm{~m}^{-2} \mathrm{~s}^{-0.5} \mathrm{~K}^{-1}\right)$ to match the observations. Our most likely solution can be summarized as: $\lambda_{\text {ecl }}=73^{\circ}, \beta_{\text {ecl }}=-62^{\circ}, P_{\text {sid }}=7.63 \pm 0.01 \mathrm{~h}$. Figure $3(\mathrm{left}$, middle) shows the model-ID 7_1, as seen from Spitzer during the IRS-observations and for the two thermal inertias marked in Fig. 2 with boxes. The match between model "7_1" with observed lightcurves is shown in Fig. 1.

We also analysed the thermal data set against the Abe et al. (2008) spin-pole solutions discussed above. The corresponding $\chi^{2}$-minima are more than a factor five worse than our two best models above (see dashed lines in Fig. 2). Some of these solutions are compatiple with the inertia range given by Campins et al. (2009) and even produced an excellent match to the IRSspectrum. Nevertheless, these solutions can be excluded with very high confidence:

(i) the match to the rest of the data set (Akari and ground-based data) is very poor (reflected in the high $\chi^{2}$-values);

(ii) the corresponding shape models are unphysical with elongations along the spin axis. Such rotational states would not be stable. The best of these models (in terms of $\chi^{2}$-minima) is shown in Fig. 3 on the right side (with the rotation along the $z$-axis with the object's largest extension).

\subsection{Thermal inertia and surface roughness}

The thermal inertia is clearly a key parameter when modelling the mid-IR data for NEAs; it strongly influences the shape of the spectral energy distribution (SED). This can be seen in the Spitzer IRS spectrum, especially in the Wien-part of the spectrum. But the thermal inertia also drives the thermal behaviour as a function of phase angle (e.g., Müller 2002), the thermal phase curve. The temperature of the unilluminated fraction of the surface changes strongly with thermal inertia. In Fig. 3 the unilluminated fraction has just rotated out of the solar insolation. In one case (left) we assumed a low thermal inertia of $\sim 20 \mathrm{~J} \mathrm{~m}^{-2} \mathrm{~s}^{-0.5} \mathrm{~K}^{-1}$, which was found to be typical for large MBAs (Müller \& Lagerros 2002), while in the second case (middle) the thermal inertia is $1000 \mathrm{~J} \mathrm{~m}^{-2} \mathrm{~s}^{-0.5} \mathrm{~K}^{-1}$, close to the value found by Müller et al. (2005) for 25143 Itokawa.

The importance of the thermal inertia in the modelling is also visible in the $\chi^{2}$-solutions (Figs. 2 and 4): the $\chi^{2}$-values change significantly when going through the whole grid of physically meaningful thermal inertias.

But Fig. 4 demonstrates that thermal inertia and surface roughness are not easy to disentangle, at least on the basis of this data set. Both parameters influence the short-wavelength SED-part where the hottest surface temperatures dominate the SED-shape. One way of solving for both parameters would be to obtain data with a larger phase angle coverage and larger wavelength coverage. The roughness plays a much bigger role at small phase angles (beaming effect), but thermal data close to opposition are not available for 162173 (1999 JU3). The thermal inertia is more important at large phase angles and at longer wavelengths where the disk-averaged temperature dominates the SED shape. In general, the larger the coverage in phase angle and wavelength, the more accurate is the determination of diameter, albedo, thermal inertia and roughness.

Figure 4 also shows that similarly low $\chi^{2}$-values can be reached independent of the surface roughness. This demonstrates that roughness effects are still important for the interpretation of thermal data (via the thermal phase curves), even at these relatively large phase angles (Müller 2002). But the data set does not allow us to constrain the surface roughness which broadens the possible range of thermal inertias as can be seen in Fig. 4. Based on our three best shape/spin-vector solutions and considering the uncertainties due to roughness, we conclude that the thermal inertia is in the range $200-600 \mathrm{~J} \mathrm{~m}^{-2} \mathrm{~s}^{-0.5} \mathrm{~K}^{-1}$. This range is in agreement with the lower limit of $150 \mathrm{~J} \mathrm{~m}^{-2} \mathrm{~s}^{-0.5} \mathrm{~K}^{-1}$ given by Campins et al. (2009) but lower than their best fit value of $700 \pm 200 \mathrm{~J} \mathrm{~m}^{-2} \mathrm{~s}^{-0.5} \mathrm{~K}^{-1}$. Our value is about a factor of two lower than the one found for 25143 Itokawa (Müller et al. 2005). We expect that the surface of 162173 (1999 JU3) is therefore different in the sense that there might be fewer rocks and boulders and that the surface includes millimetre sized particles (as opposed to the $\mathrm{cm}$-sized gravel on Itokawa). We also find a rigorous lower limit to the thermal inertia of about $100 \mathrm{~J} \mathrm{~m}^{-2} \mathrm{~s}^{-0.5} \mathrm{~K}^{-1}$, similar to Campins et al. (2009). This limit is not compatible with a thick dusty regolith covering the entire surface which would result in a very low thermal conductivity and thermal inertia values well below $100 \mathrm{~J} \mathrm{~m}^{-2} \mathrm{~s}^{-0.5} \mathrm{~K}^{-1}$, which would in principle be possible for an object of that size and the relativly slow rotation rate. For comparison, the Moon's thick regolith gives a value below $40 \mathrm{~J} \mathrm{~m}^{-2} \mathrm{~s}^{-0.5} \mathrm{~K}^{-1}$ (Keihm 1984, calculated for $T=300 \mathrm{~K})$.

\subsection{Radiometric diameter and albedo solution}

Our best fit to all observations, as represented in Fig. 2 by the solid line, resulted in a radiometric effective diameter of $0.87 \pm 0.02 \mathrm{~km}$ and $0.070 \pm 0.003$ for the visual geometric albedo. Both values are within the error bars of the solutions found by Hasegawa et al. (2008) and by Campins et al. (2009), but now with much smaller errors. The uncertainties are based on the best $\chi^{2}$-values for model-IDs "7_1" and " $8 \_2$ " and the full variation in roughness (as shown in Fig. 4). The radiometric effective diameter is connected to the most likely shape model and spin-vector solution from above and corresponds to the size of a 

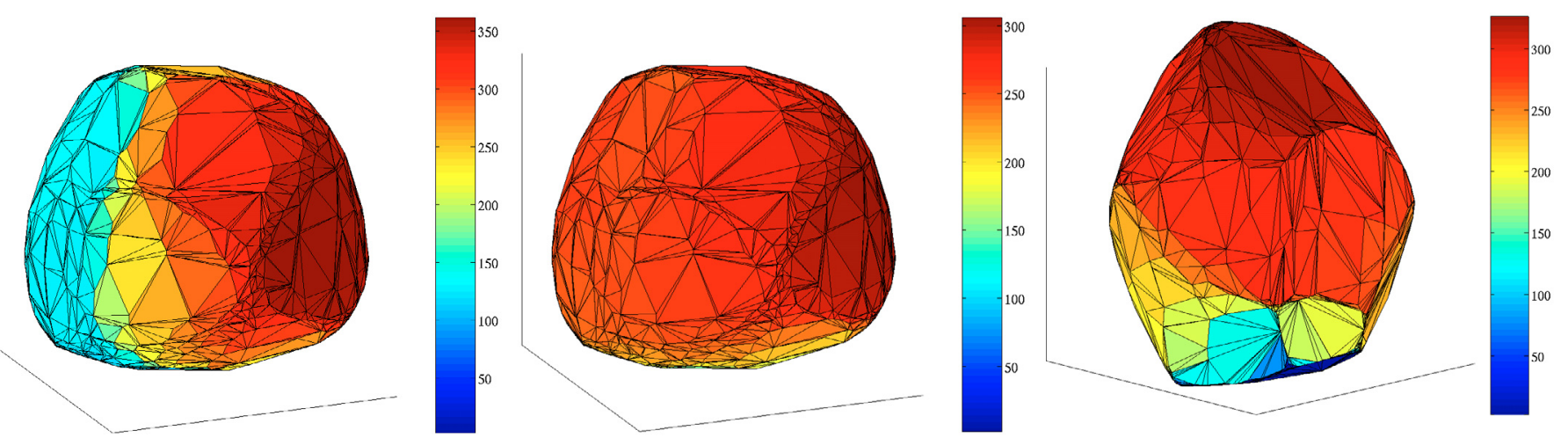

Fig. 3. Left and middle: TPM implementation of the shape model 7_1 at the time of the Spitzer IRS observations on 2008-May-02 02:01 UT, as seen from Spitzer in asteroid-coordinates, i.e., the z-axis goes along the rotation axis. Left: low thermal inertia. Middle: high thermal inertia. Both solutions are marked with squares in Fig. 2. Right: shape model fixed on the Abe et al. (2008) spin-vector and tuned to match the Campins et al. (2009) findings. The rotation along the largest object extension (z-axis) is unphysical. The surface temperatures are given in Kelvin.

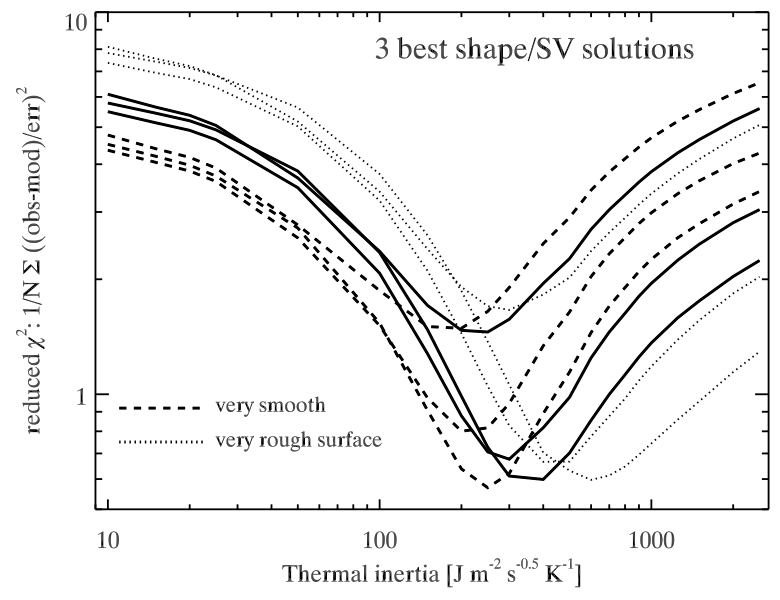

Fig. 4. TPM $\chi^{2}$-optimization process for the shape and spin-axis solutions with the lowest $\chi^{2}$-values (models 7_1, 8_2, 14_8). The solid lines are the "default roughness" values derived for MBAs.

spherical object with equal volume. Using the determined possible thermal inertia range of $200-600 \mathrm{~J} \mathrm{~m}^{-2} \mathrm{~s}^{-0.5} \mathrm{~K}^{-1}$ and uncertainties in roughness and $H_{\mathrm{V}}$-magnitude lead to a possible value for the effective diameter of $0.87 \pm 0.03 \mathrm{~km}$. The derived geometric albedo provides the best solution between all thermal observations over the full wavelength and phase angle range and the absolute $H_{\mathrm{V}}$-magnitude. The $H_{\mathrm{V}}$-magnitude was given with only 0.02 mag error. Using a more realistic $H_{\mathrm{V}}$-error of $\pm 0.1 \mathrm{mag}$ leads to a final geometric albedo value of $0.070 \pm 0.006$. The small uncertainties reflect the importance of multi-epoch, multiwavelength and large phase angle coverage for thermophysical studies of small bodies. In a similar study by Müller et al. (2005), also based on a large thermal data set and a shape model from lightcurve inversion techniques, the derived effective diameter agreed within $2 \%$ of the true, in-situ diameter (Müller et al. 2005; Fujiwara et al. 2006). The quoted uncertainties above are formal errors from the $\chi^{2}$ optimization, including the possible range in thermal inertia, roughness and $H_{\mathrm{V}}$.

The remaining discrepancy between the Spitzer and the Akari-flux at $15 \mu \mathrm{m}$ (seen in Fig. 5 bottom) might be related to an additional error introduced by the flux scaling done by Campins et al. (2009) to match the short-wavelength part of the spectrum $(<14 \mu \mathrm{m})$ to the long-wavelength part of the spectrum $(>14 \mu \mathrm{m})$.
The mismatch is caused mainly by the placement of the object within the IRS slit and reflected in the specified $10 \%$ systematic absolute calibration uncertainty.

Although the Fig. 4 solutions and the model match in Fig. 5 look very convincing, there are some uncertainties remaining: The spin-vector and shape solution from lightcurve inversion techniques is not very robust; more lightcurve observations are needed to improve the quality. The $\chi^{2}$-test works best if the thermal observations cover a wide range of wavelengths, phase angles (before and after opposition) and rotational phases. But all thermal observations have been taken at pre-opposition (positive) phase angles (leading the Sun) where the unilluminated part of the surface visible to the observer is either warm (prograde rotation) or cold (retrograde rotation); observations after opposition are not yet available. A combined before and after opposition data set would constrain the sense of rotation and the thermal properties much better, observations close to opposition would determine the surface roughness a bit better, hence constrain the thermal inertia further.

It is also important to note here that higher thermal inertias ( $>600 \mathrm{~J} \mathrm{~m}^{-2} \mathrm{~s}^{-0.5} \mathrm{~K}^{-1}$, Fig. 3 middle) would make a slightly better fit to the Spitzer IRS spectrum (improvement mainly at the shortest wavelengths in Fig. 5 bottom), but would cause a significant dependency in the diameter and albedo solutions with phase angle. The measurements taken at around $20^{\circ}$ phase angle (Subaru) would then have fluxes that are about $40 \%$ higher than the corresponding model predictions (i.e., values $>1.4$ in Fig. 5, top). The Akari fluxes would still be $\sim 20 \%$ higher than the model predictions. Our observational data set covering about $30^{\circ}$ in phase angle constrains the possible thermal inertia to values below $700 \mathrm{~J} \mathrm{~m}^{-2} \mathrm{~s}^{-0.5} \mathrm{~K}^{-1}$.

\section{Conclusions}

The radiometric analysis provides the following results: (i) a strong indication of a retrograde sense of rotation; (ii) a spinvector with $\lambda_{\text {ecl }}=73^{\circ}, \beta_{\text {ecl }}=-62^{\circ}, P_{\text {sid }}=7.63 \pm 0.01 \mathrm{~h}$ and $\gamma_{0}=0$ at $T_{0}=2454289.0$, derived for the first time based on a combined analysis of visual lightcurve data and thermal observations; (iii) a shape model (here labelled with 7_1) as shown in Fig. 3 (left \& middle); (iv) a thermal inertia in the range 200 to $600 \mathrm{~J} \mathrm{~m}^{-2} \mathrm{~s}^{-0.5} \mathrm{~K}^{-1}$; (v) a radiometric effective diameter (of an equal volume sphere) of $D_{\text {eff }}=0.87 \pm 0.03 \mathrm{~km}$; (vi) a radiometric geometric albedo of $p_{V}=0.070 \pm 0.006$; (vii) a lower thermal inertia than for Itokawa, suggesting the presence 

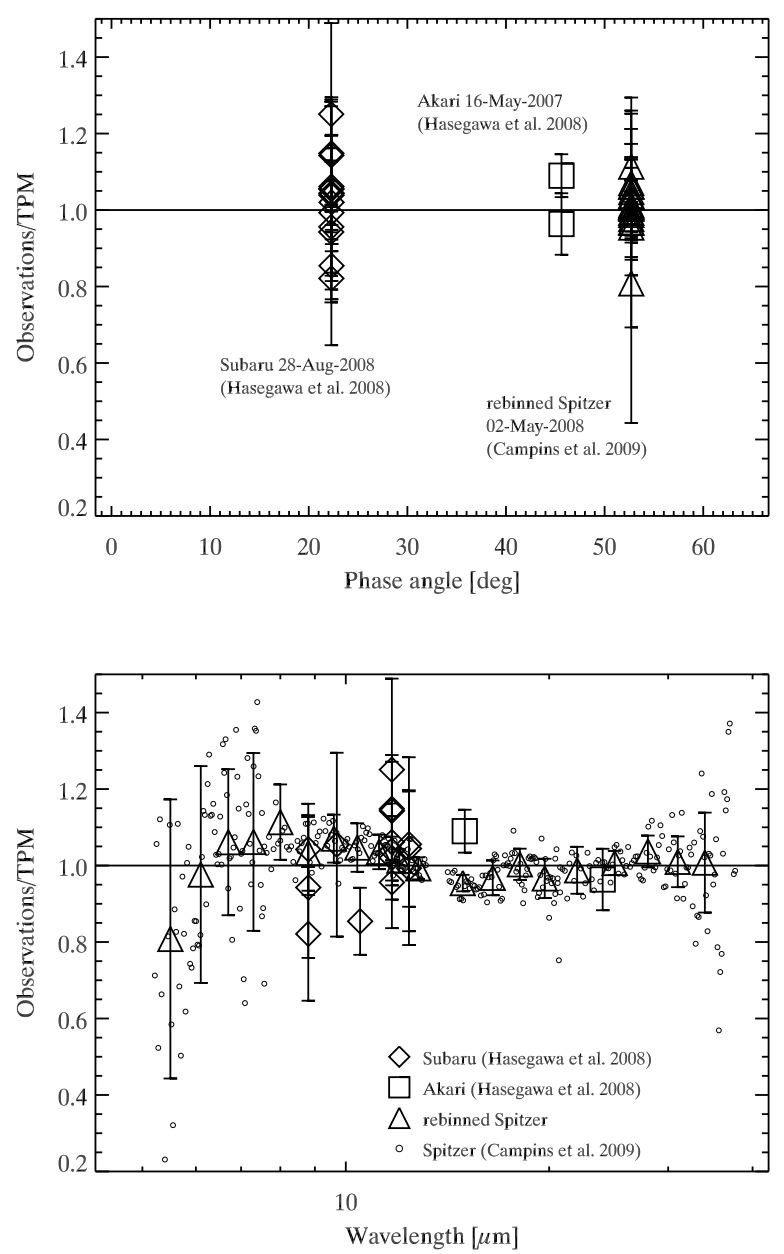

Fig. 5. All observations divided by the corresponding TPM predictions based on our optimized radiometric solution. Top: as a function of phase angle. Bottom: as a function of wavelength. The full set of Spitzer IRS data are shown with little circles (Campins et al. 2009), the triangles are the re-binned data, the Akari IRC data are represented by squares; the Subaru-COMICS observations by diamond symbols.

of smaller particles, $<\mathrm{cm}$-sized, in the regolith, though likely not fine dust; (viii) very good agreement in the radiometric solutions between the Spitzer, the Akari and the Subaru observations; (ix) an excellent match of the flux changes with phase angle (the phase angle range covered here is from $\sim 20^{\circ}$ to $\sim 55^{\circ}$ ).

The example of 162173 (1999 JU3) shows that a combination of visual lightcurves (reflected sunlight) and mid/far-IR photometry or photo-spectroscopy (thermal emission) can improve the quality of shape and spin-vector solutions significantly.

\begin{abstract}
Acknowledgements. J. D. received grants from the Czech Science Foundation (GACR P209/10/0537) and the Research Program MSM0021620860 of the Ministry of education. S. H. was supported by the Space Plasma Laboratory, ISAS, JAXA. We are also grateful to Professor N. Kawai and the gamma-ray bursts project members for furnishing their optical camera at Ishigakijima Astronomical Observatory. Development of the optical CCD camera at Ishigakijima Astronomical Observatory was supported by the Ministry of Education, Science, Sports and Culture, Grant-in-Aid for Creative Scientific Research. T.K. thanks to the JSPS Research Fellowships for Research Abroad for their financial support. This work was supported in part by the NASA Planetary Astronomy Program and performed in part at the Jet Propulsion Laboratory. We thank the Steward Observatory of the University of Arizona for its allocation of telescope time. We also thank the referee Dr. J. Emery for very helpful comments.
\end{abstract}

\section{References}

Abe, M., Kawakami, K., Hasegawa, S., et al. 2008, COSPAR Scientific Assembly, B04-0061-08

Binzel, R. P., Perozzi, E., Rivkin, A. S., Rossi, A., et al. 2004, Meteorit. Planet. Sci., 39, 351

Campins, H., Emery, J. P., Kelley, M., et al. 2009, A\&A, 503, L17

Fujiwara, A., Kawaguchi, J., Uesugi, K., et al. 2006, Science, 312, 1330

Hasegawa, S., Müller, T. G., Kawakami, K., et al. 2008, PASJ, 60, 399

Kaasalainen, M., \& Torppa, J. 2001, Icarus, 153, 24

Keihm, S. J. 1984, Icarus, 60, 568

Lagerros, J. S. V. 1996, A\&A, 310, 1011

Lagerros, J. S. V. 1997, A\&A, 325, 1226

Lagerros, J. S. V. 1998, A\&A, 332, 1123

Magnusson. P. 1986, Icarus, 68, 1

Müller, T. G., \& Lagerros, J. S. V. 1998, A\&A, 338, 340

Müller, T. G., Lagerros, J. S. V., Burgdorf, M., et al. 1999, in The Universe as Seen by ISO, ed. P. Cox, \& M. F. Kessler, ESA SP-427, 141

Müller, T. G., \& Lagerros, J. S. V. 2002, A\&A, 381, 324

Müller, T. G. 2002, M\&PS, 37, 1919

Müller, T. G., Sekiguchi, T., Kaasalainen, M., Abe, M., \& Hasegawa, S. 2005, A\&A, 443, 347 (M05)

Mueller, M. 2007, DLR, Thesis FU Berlin (http://www.diss.fu-berlin. de/diss/receive/FUDISS_thesis_000000002596) 